\title{
DYNAMIC ANALYSIS OF A COUPLED FLUID STRUCTURE PROBLEM WITH FLUID SLOSHING
}

\author{
Jean François SIGRIST \\ Service Scientifique et Technique \\ DCN Propulsion \\ 44620 LA MONTAGNE, France \\ jean-francois.sigristeden.fr
}

\author{
Christian LAINE \\ Service Scientifique et Technique \\ DCN Propulsion \\ 44620 LA MONTAGNE, France
}

\author{
Bernard PESEUX \\ Laboratoire de Mécanique et Matériaux \\ Division Mécanique des Structures \\ Ecole Centrale de Nantes - 1, rue de La Noë \\ 44321 Nantes Cedex 3, France
}

\begin{abstract}
The present paper is related to the study of a generic linear coupled fluid/structure problem, in which an elastic beam is coupled with an inviscid fluid, with or without sloshing effects. A previous study [18] focussed on added mass effects; the present study is devoted to the coupling effects between fluid sloshing modes and structure with fluid added mass modes.

The discretization of the coupled linear equations is performed with an axisymmetric fluid pressure formulated element, expanded in terms of a FOURIER series [14]. Various linear fluid model are taken into account (compressible, uncompressible, with or without sloshing) with the corresponding coupling matrix operator. The modal analysis is performed with a MATLAB program, using the non-symmetric LANCZOS algorithm [16]. The temporal analysis is performed with classical numerical techniques [10], in order to describe the dynamic response of the coupled problem subjected to a simple sine wave shock.
\end{abstract}

The coupling effects are studied in various conditions represented by several non-dimensionnal numbers [12] such as the dynamic FROUDE number and the mass number, based on the geometrical and physical characteristics of the coupled problem.

Comparisons are performed on the coupled problem with or without free surface modeling, with a modal and temporal analysis. Coupling effects are exhibited and quantified; the numerical results obtained in the modal analysis here are in good agreement with other previous studies, carried out on different geometry $[3,15]$. The temporal analysis gives another point of view on the importance of the coupling effects and their importance at low dynamic FROUDE numbers.

The present study gives and will be completed with a non-linear analysis (for both fluid and structure problems) of the coupled problem, using a finite element and finite volume explicit coupling procedure [19].

\section{INTRODUCTION}

The numerical simulation of coupled fluid structure problems is a subject of great interest, and many studies were carried out on this topic over the past years [13], among which the influence of a fluid free surface on the dynamic of the coupled problem (see e.g. in some recent studies $[1,6,17])$. In the present paper, we study the following generic coupled problem, represented by Fig. (1), and focus on the coupling effect occurring between the fluid sloshing modes and the bending structure modes.

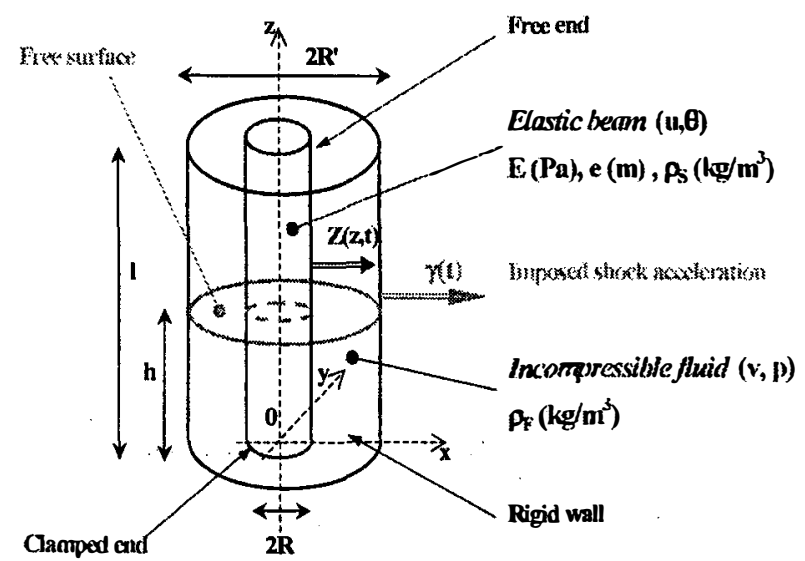

Figure 1. Generic coupled fluid structure problem with fluid free surface 
The problem is characterized by various non-dimensionnal numbers such as the confinement ratio $\alpha=\frac{R^{\prime}}{R}$, the filling ratio $\lambda=\frac{l}{h}$ and the length ratio $\eta=\frac{l}{R} ;$ the dynamic FROUDE number is defined as: $F_{D}=\frac{e}{R} \times \sqrt{\frac{E}{\rho_{s} g l}}$.

\section{GOVERNING EQUATIONS AND VARIATIONNAL FORMULATION FOR THE DYNAMIC PROBLEM}

The structure problem is described in terms of displacement; the governing equations are the following ones (the description includes the dynamic equation, the structure boundary conditions and the coupling condition with the fluid problem):

$$
\begin{array}{cc}
\rho_{S} S \frac{\partial^{2} u}{\partial t^{2}}+E I \frac{\partial^{4} u}{\partial z^{4}}=f-\int_{0}^{2 \pi} p(R, \theta, t) R \cos \theta d \theta \\
u_{(z=0)}=0 & u_{(z=l)}=0 \\
\frac{\partial u_{(z=0)}}{\partial z_{(z)}}=0 & \frac{\partial u}{\partial z}=0
\end{array}
$$

The fluid problem is described in terms of pressure [14]; the governing equations are (the description includes the local equation, the fluid boundary conditions and the coupling condition with the structure):

$$
\begin{gathered}
\Delta p=0 \\
\frac{\partial p}{\partial z}_{(z=0)}=\frac{\partial p}{\partial r}=0 \\
\frac{\partial p}{\partial r=R)}_{(r=R)}=-\rho_{F} \frac{\partial^{2} u}{\partial t^{2}} \cos \theta
\end{gathered}
$$

The free surface condition can be described with or without gravity waves, that is:

$$
p_{(z=h)}=0
$$

in the former case and:

$$
{\frac{\partial p}{\partial z_{(z=h)}}}=-\frac{1}{g}{\frac{\partial^{2} p}{\partial z^{2}}}_{(z=h)}
$$

in the latter case.

The variationnal formulation of the dynamic problem is the following one; find $u(t)$ and $p(t)$ such as for all admissible virtual fields of displacement and pressure $(\delta u, \delta p)$ :

$$
\begin{aligned}
& \frac{d^{2} m_{S}(u(t), \delta u)}{d t^{2}}+k_{S}(u(t), \delta u)= \\
& <f(t), \delta u>-r^{*}(p(t), \delta u) \\
& \frac{d^{2} m_{F}(p(t), \delta p)}{d t^{2}}+k_{F}(p(t), \delta p)= \\
& \rho_{F} \frac{d^{2} r(u(t), \delta p)}{d t^{2}}
\end{aligned}
$$

with the initial conditions for the pressure and displacement field :

$$
\begin{array}{cc}
u(0)=u_{0} & p(0)=p_{0} \\
\frac{\partial u}{\partial t}(0)=\dot{u}_{0} & \frac{\partial p}{\partial t}(0)=\dot{p}_{0}
\end{array}
$$

The various linear and bilinear forms used in the above formulation are the following ones.

- $m_{s}(u, \delta u)=\int_{0}^{h} \rho_{s} S u(z) \delta u(z) d z$ is the structure mass bilinear form, $\quad k_{s}(u, \delta u)=\int_{0}^{h} E I \frac{d^{2} u(z)}{d z^{2}} \frac{d^{2} \delta u(z)}{d z^{2}} d z$ is the structure stiffness bilinear form;

- $\quad m_{F}^{\prime}(p, \delta p)=\int_{R}^{R^{\prime}} \int_{0}^{2 \pi} \frac{p(r, \theta, h) \delta p(r, \theta, h)}{g} r d r \cos \theta d \theta$ and $k_{F}(p, \delta p)=\int_{R}^{R^{\prime}} \int_{0}^{2 \pi} \int_{0}^{h} \nabla p(r, \theta, z) \nabla \delta p(r, \theta, z) r d r \cos \theta d \theta d z$ are the fluid mass and stiffness bilinear forms ;

- $\quad r(p, u)=-\int_{0}^{h} \int_{0}^{2 \pi} p(R, \theta, z) R \cos \theta d \theta u(z) d z$ is the fluid/structure interaction operator, $r^{*}(.,$.$) is the adjoint operator.$

\section{FINITE ELEMENT DISCRETIZATION}

The discretization of the variationnal formulation is performed with a finite element approach. Since the coupled problem is geometrically axi-symmetric but the dynamic loading is non axisymmetric, the pressure is expanded into a FOURIER serie as ${ }^{l}$ :

$$
p(r, \theta, z)=p_{0}(r, z)+\sum_{n \geq 1} p_{n}(r, z) \cos (n \theta)+\sum_{m \geq 1} p_{m}(r, z) \sin (m \theta)
$$

The 3D fluid problem $(r, \theta, z)$ is then turned into a $2 \mathrm{D}$ problem $(r, z)$. The two-dimensionnal fluid domain is meshed with four nodes linear finite element. Figure (2) gives a representation of the fluid reference element.

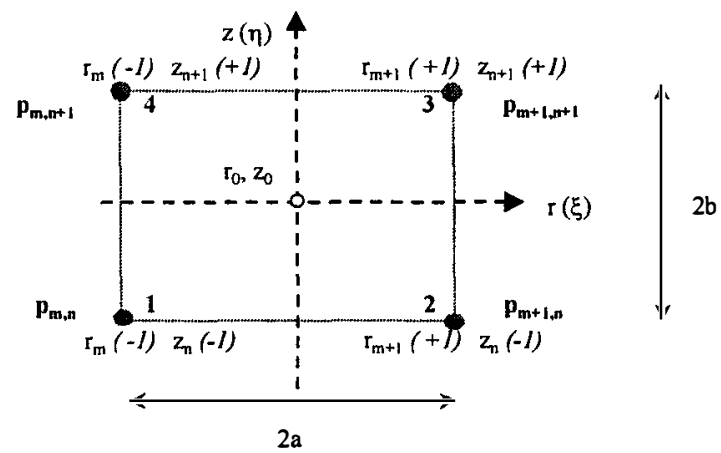

Figure 2. Linear 2D fluid finite element

\footnotetext{
${ }^{1}$ In practical application with the beam coupling, only the first cosine term $(n=1)$ is to be taken into account.
} 
The fluid shape functions are linear with respect to both local variables $\xi, \boldsymbol{\eta}$ :

$$
N_{i}^{F}(\xi, \eta)=\frac{\left(1+\xi_{i} \xi\right)\left(1+\eta_{i} \eta\right)}{4}
$$

The discretization of the fluid bilinear forms gives the mass and stiffness matrices $M^{\prime}{ }_{F}, K_{F}=\bar{K}_{F}+n^{2} \widetilde{K}_{F}$ for the fluid problem. The corresponding elementary matrices are defined by the following analytical expressions.

$$
\begin{aligned}
& \bar{k}_{i j}^{F}=a b r_{0}\left[\frac{\xi_{i} \xi_{j}}{4 a^{2}}\left(1+\frac{\eta_{i} \eta_{j}}{3}\right)+\frac{\eta_{i} \eta_{j}}{4 a^{2}}\left(1+\frac{\xi_{i} \xi_{j}}{3}\right)\right]+\frac{a^{2} \eta_{i} \eta_{j}}{12 b}\left(\xi_{i}+\xi_{j}\right) \\
& \tilde{k}_{i j}^{F}=\frac{b}{8}\left[1+\frac{\eta_{i} \eta_{j}}{3}\right] \times \\
& {\left[2\left(\xi_{i}+\xi_{j}\right)-\frac{2 r_{0} \xi_{j} \xi_{j}}{a}+\left(1-\frac{r_{0}\left(\xi_{i}+\xi_{j}\right)}{a}+\frac{r_{0}^{2} \xi_{i} \xi_{j}}{a^{2}}\right) \times \ln \left(\frac{r_{0}+a}{r_{0}-a}\right)\right]} \\
& m_{i j}^{\prime F}=\frac{a}{g}\left[r_{0}+\frac{r_{0} \xi_{i} \xi_{j}}{3}+\frac{a\left(\xi_{i}+\xi_{j}\right)}{3}\right] \times\left[\left(1+\eta_{i}\right)\left(1+\eta_{j}\right)\right]
\end{aligned}
$$

If compressibility effects are taken into account in the fluid model, the dynamic equation of the fluid problem is $\frac{1}{c^{2}} \cdot \frac{\partial^{2} p}{\partial t^{2}}-\Delta p=0$. The variationnal formulation for the fluid problem has to take into account the mass bilinear form $m_{F}(p, \delta p)=\int_{R}^{R^{\prime}} \int_{0}^{2 \pi h} \int_{0}^{h} \frac{p(r, \theta, z) \delta p(r, \theta, z)}{c^{2}} r d r \cos \theta d \theta d z$, which is discretized into the mass matrix $M_{F}$. The corresponding elementary matrix is :

$$
m_{i j}^{F}=\frac{a b}{4}\left[r_{0}+\frac{r_{0} \xi_{j} \xi_{j}}{3}+\frac{a\left(\xi_{i}+\xi_{j}\right)}{3}\right] \times\left[1+\frac{\eta_{i} \eta_{j}}{3}\right]
$$

The discretization of the structure problem is performed with two node finite element with two degrees of freedom $u, \partial u / \partial z$ and cubic shape functions [5].

The coupled dynamic fluid/structure problem is then written in the non-symmetric form:

$$
\left[\begin{array}{cc}
M_{S} & 0 \\
-\rho_{F} R^{T} & M_{F}^{*}
\end{array}\right]\left\{\begin{array}{l}
\ddot{U} \\
\ddot{P}
\end{array}\right\}+\left[\begin{array}{cc}
K_{S} & R \\
0 & K_{F}
\end{array}\right]\left\{\begin{array}{l}
\ddot{U} \\
\ddot{P}
\end{array}\right\}=\left\{\begin{array}{l}
\phi_{S} \\
\phi_{F}
\end{array}\right\}
$$

where $R$ is the coupling matrix, which discretizes $r(.,$.$) .$

When compressibility effects are taken into account in the fluid problem, he coupled problem is:

$$
\left[\begin{array}{cc}
M_{S} & 0 \\
-\rho_{F} R^{T} & M_{F}+M_{F}^{\prime}
\end{array}\right]\left\{\begin{array}{l}
\ddot{U} \\
\ddot{P}
\end{array}\right\}+\left[\begin{array}{cc}
K_{S} & R \\
0 & K_{F}
\end{array}\right]\left\{\begin{array}{l}
U \\
P
\end{array}\right\}=\left\{\begin{array}{l}
\phi_{S} \\
\phi_{F}
\end{array}\right\}
$$

A MATLAB program is developed in order to perform the numerical analysis. This program is validated with the comparison between calculated eigenvalues of a fluid problem with free surface and analytical eigenvalues.

\begin{tabular}{ccccc}
\hline Frequency & $\mathbf{f}_{\mathbf{1}}$ & $\mathbf{f}_{\mathbf{2}}$ & $\mathbf{f}_{\mathbf{3}}$ & $\mathbf{F}_{\mathbf{4}}$ \\
\hline Analytical & 0.9963 & 2.8519 & 3.9734 & 4.8513 \\
Numerical & 0.9965 & 2.8641 & 4.0398 & 5.0367 \\
\hline
\end{tabular}

Table 1. Elementary validation of the fluid finite element for sloshing modes in axi-symmetric problems

Table (1) gives the analytical and numerical frequencies (in $\mathrm{Hz}$ ) for the elementary problem ${ }^{2}$.

\section{MODAL ANALYSIS OF THE COUPLED PROBLEM}

The modal analysis of the coupled problem cannot be performed with the mass and stiffness matrix given in Eq. (13) because the matrix $M_{F}^{\prime}$ is singular. One has to split the degrees of freedom of the fluid problem according to $\{P\}=\left\{\begin{array}{c}P_{O} \\ \widetilde{P}\end{array}\right\}$ where $P_{O}$ are the degrees of freedom for the fluid free surface and $\widetilde{P}$ are the degrees of freedom of the rest of the fluid. Equation () becomes:

$$
\left[\begin{array}{ccc}
M_{S} & 0 & 0 \\
-\rho_{F} R_{o}{ }^{r} & M_{F}^{o} & 0 \\
-\rho_{F} R^{T} & 0 & 0
\end{array}\right]\left\{\begin{array}{c}
\ddot{U} \\
\ddot{P} \\
\ddot{\widetilde{P}}
\end{array}\right\}+\left[\begin{array}{ccc}
K_{S} & R_{o} & \widetilde{R} \\
0 & K_{F}^{o} & \widetilde{K}_{F}^{o} \\
0 & \widetilde{K}_{F}^{o r} & \widetilde{K}_{F}
\end{array}\right]\left\{\begin{array}{c}
U \\
P_{o} \\
\widetilde{P}
\end{array}\right\}=\left\{\begin{array}{c}
\phi_{S} \\
\phi_{O} \\
\widetilde{\phi}
\end{array}\right\}
$$

After static condensation, the system is reformulated in terms of $U$ and $P_{o}$. The corresponding modal problem is then:

$$
\begin{aligned}
& {\left[\begin{array}{cc}
K_{S} & R_{o}-\widetilde{R}_{K}^{-1} \widetilde{K}_{F}^{o T} \\
0 & K_{F}^{o}-\widetilde{K}_{F}^{o T} \widetilde{K}_{F}^{-1} \widetilde{K}_{F}^{o}
\end{array}\right]\left\{\begin{array}{l}
U(\omega) \\
P_{o}(\omega)
\end{array}\right\}=} \\
& \omega^{2}\left[\begin{array}{cc}
M_{S}+\rho_{F} \widetilde{R}_{F} \widetilde{K}^{-1} \widetilde{R}^{T} & 0 \\
-\rho_{F}\left(R_{o}^{T}-\widetilde{K}_{F}^{o} \widetilde{K}_{F}^{-1} \widetilde{R}^{T}\right) & M_{F}^{o}
\end{array}\right]\left\{\begin{array}{l}
U(\omega) \\
P_{o}(\omega)
\end{array}\right\}
\end{aligned}
$$

The coupled problem has the same structure as the initial problem given by Eq. (13): the non-symmetry is typical of a displacement/pressure formulation $[14,16]$. Equation (16) also exhibits the coupling matrix between the fluid and structure problem $R_{O}-\widetilde{R} \widetilde{K}_{F}{ }^{-1} \widetilde{K}_{F}^{o^{T}}$, which takes into account the free surface terms. On the other hand, the structure part of the coupled problems exhibits the hydrodynamic added mass term $\rho_{F} \widetilde{R} \widetilde{K}_{F}{ }^{-1} \widetilde{R}^{T}$, which correspond to the structure problem coupled with the uncompressible fluid with boundary condition $p=0$ for the free surface. Thus, restricting Eq. (16) to the structure or fluid separate terms leads to the determination of the eigenmodes of:

$\checkmark$ the fluid sloshing without structure coupling effects the problem to solve is:

$$
\left(K_{F}^{o}-\widetilde{K}_{F}^{o^{T}} \widetilde{K}_{F}^{-1} \widetilde{K}_{F}^{o}\right) \times P_{o}(\omega)=\omega^{2} M_{F}^{o} \times P_{o}(\omega)
$$

\footnotetext{
${ }^{2}$ The validation case is that of a cylindrical tank for which the analytical eingenvalues are given by [8], p. 288. Table (1) gives the comparison of numerical and analytical model with $R=0.1 \mathrm{~m}, \eta=1, \alpha=2$ and $g=10 \mathrm{~m} / \mathrm{s}^{2}$. The mode order is $n=1$.
} 
$\checkmark \quad$ the structure problem coupled with the fluid without fluid sloshing effects; the problem to solve is:

$$
K_{S} \times U(\omega)=\omega^{2}\left(M_{S}+\rho_{F} \tilde{R} \widetilde{K}_{F}{ }^{-1} \widetilde{R}^{r}\right) \times U(\omega)
$$

The non-symmetric problem given by Eq. (16) is solved using the LANCZOS algorithm adapted to non-symmetric problems [16]. Table (2) compares the computed eignevalues with the lanczos function developed in the MATLAB code and the theorical eigenvalues for the non-symmetric example proposed in [16]; this comparison validates the numerical calculation of eigenvalues for generalized non-symmetric problem with our numerical code.

\begin{tabular}{cc}
\hline Theorical eigenvalues & MATLAB lanczos function \\
\hline $0.10000+0.00000 i$ & $0.1000+0.0000 i$ \\
$4.00000 \pm 1.00000 i$ & $4.0000 \pm 1.0000 i$ \\
$2.40000 \pm 7.32000 i$ & $2.39974 \pm 7.31897 i$ \\
$7.00000+0.00000 i$ & $6.99999+0.00000 i$ \\
$7.00000+0.00000 i$ & $7.00088+0.00000 i$ \\
$8.00000 \pm 127.00000 i$ & $7.99997 \pm 127.00001 i$ \\
$11.00000+0.00000 i$ & $11.00005+0.00000 i$ \\
$130.000000+0.00000 i$ & $129.99998+0.00000 i$ \\
$-2000.00000+0.00000 i$ & $-1999.99995+0.00000 i$ \\
\hline
\end{tabular}

Table 2. Elementary validation of the lanczos function implemented in the Matlab code

A modal analysis of decoupled and coupled fluid/structure problem is performed on the generic case represented by Fig. (1). The geometrical and physical data of the problem are characterized by the non-dimensionnal numbers $\alpha=2, \eta=5, \lambda, F_{D}$ and $M_{A}$. Table (3) gives the eigenfrequencies of the structure without fluid coupling.

\begin{tabular}{lcccc}
\hline \multicolumn{1}{c}{ Frequency $(\mathrm{Hz})$} & $\mathbf{f}_{\mathbf{1}}$ & $\mathbf{f}_{\mathbf{2}}$ & $\mathbf{f}_{\mathbf{3}}$ & $\mathbf{f}_{\mathbf{4}}$ \\
\hline Structure mode $\left(F_{D} \sim 1\right)$ & 3.4705 & 21.7493 & 60.90 & 119.34 \\
Structure mode $\left(F_{D} \sim 10\right)$ & 34.71 & 217.49 & 608.98 & 1193.4 \\
\hline
\end{tabular}

Table 3. Structure mode for $F_{D} \sim 100$ and $F_{D}=10$

Tables (4) to (7) show the eigenfrequencies of the fluid problem with and without structure coupling, the structure problem coupled with fluid with or without fluid sloshing, with $F_{D}=100$ and $M_{A}=8$, for different values of $\lambda$.

\begin{tabular}{lcccc}
\hline \multicolumn{1}{c}{ Frequency $(\mathrm{Hz})$} & $\mathbf{f}_{\mathbf{1}}$ & $\mathbf{f}_{\mathbf{2}}$ & $\mathbf{f}_{\mathbf{3}}$ & $\mathbf{f}_{\mathbf{4}}$ \\
\hline Fluid w/o structure & 1.0996 & 2.8757 & 4.0789 & 5.1344 \\
Structure w/o sloshing & 34.6724 & 212.768 & 555.541 & 1046.20 \\
Fluid with structure & 1.0996 & 2.8757 & 4.0789 & 5.1344 \\
Structure with sloshing & 34.6729 & 212.770 & 555.543 & 1046.20 \\
\hline
\end{tabular}

Table 4. Uncoupled and couppled fluid structure mode with or without fluid sloshing for $F_{D} \sim 10, M_{A}=8$ and $\lambda=25 \%$

\begin{tabular}{lcccc}
\hline \multicolumn{1}{r}{ Frequency $(\mathrm{Hz})$} & $\mathbf{f}_{1}$ & $\mathbf{f}_{2}$ & $\mathbf{f}_{\mathbf{3}}$ & $\mathbf{f}_{4}$ \\
\hline Fluid w/o structure & 1.2546 & 2.8761 & 4.0789 & 5.1344 \\
Structure w/o sloshing & 33.6284 & 164.728 & 461.918 & 944.62 \\
Fluid with structure & 1.2545 & 2.8760 & 4.0788 & 5.1344 \\
Structure with sloshing & 33.6349 & 164.7326 & 461.918 & 944.620 \\
\hline
\end{tabular}

Table 5. Uncoupled and coupled fluid structure mode with or without fluid sloshing for $F_{D} \sim 10, M_{A}=8$ and $\lambda=50 \%$

\begin{tabular}{lcccc}
\hline \multicolumn{1}{c}{ Frequency $(\mathrm{Hz})$} & $\mathbf{f}_{\mathbf{1}}$ & $\mathbf{f}_{\mathbf{2}}$ & $\mathbf{f}_{\mathbf{3}}$ & $\mathbf{f}_{\mathbf{4}}$ \\
\hline Fluid w/o structure & 1.2905 & 2.8761 & 4.0789 & 5.1344 \\
Structure w/o sloshing & 27.2966 & 136.706 & 404.423 & 869.101 \\
Fluid with structure & 1.2899 & 2.8756 & 4.0787 & 5.1343 \\
Structure with sloshing & 27.3250 & 136.707 & 404.423 & 869.110 \\
\hline
\end{tabular}

Table 6. Uncoupled and coupled fluid structure mode with or without fluid sloshing for $F_{D} \sim 10, M_{A}=8$ and $\lambda=75 \%$

\begin{tabular}{lcccc}
\hline \multicolumn{1}{c}{ Frequency $(\mathrm{Hz})$} & $\mathbf{f}_{\mathbf{1}}$ & $\mathbf{f}_{\mathbf{2}}$ & $\mathbf{f}_{\mathbf{3}}$ & $\mathbf{f}_{4}$ \\
\hline Fluid w/o structure & 1.2962 & 2.8761 & 4.0789 & 5.1344 \\
Structure w/o sloshing & 19.3432 & 122.546 & 377.832 & 804.374 \\
Fluid with structure & 1.2943 & 2.8750 & 4.0783 & 5.1341 \\
Structure with sloshing & 19.4094 & 122.552 & 377.834 & 804.374 \\
\hline
\end{tabular}

Table 7. Uncoupled and coupled fluid structure mode with or without fluid sloshing for $F_{D} \sim 10, M_{A}=8$ and $\lambda=100 \%$

For a high dynamic FROUDE number, the fluid and structure coupled modes are practically uncoupled. The fluid eigenfrequencies are slightly increased as well as the structure eigenfrequencies with sloshing compared to the eigenfrequencies without sloshing. These observations are made for all fluid filling ratios. In this case, a separate modal analysis for fluid and structure problem can be performed.

Tables (8) to (11) show the eigenfrequencies of the fluid problem with and without structure coupling, the structure problem coupled with fluid with or without fluid sloshing, with $F_{D}=10$ and $M_{A}=8$, for different values of $\lambda$.

\begin{tabular}{lcccc}
\hline \multicolumn{1}{c}{ Frequency $(\mathrm{Hz})$} & $\mathbf{f}_{1}$ & $\mathbf{f}_{2}$ & $\mathbf{f}_{3}$ & $\mathbf{f}_{4}$ \\
\hline \hline Fluid w/o structure & 1.0996 & 2.8757 & 4.0789 & 5.1344 \\
Structure w/o sloshing & 3.4672 & 21.2768 & 55.5542 & 104.618 \\
Fluid with structure & 1.0989 & 2.8728 & 4.0798 & 5.1345 \\
Structure with sloshing & 3.4698 & 21.2952 & 55.5690 & 104.623 \\
\hline
\end{tabular}

Table 8. Uncoupled and coupled fluid structure mode with or without fluid sloshing for $F_{D} \sim 1, M_{A}=8$ and $\lambda=25 \%$

\begin{tabular}{lcccc}
\hline \multicolumn{1}{c}{ Frequency $(\mathrm{Hz})$} & $\mathbf{f}_{\mathbf{1}}$ & $\mathbf{f}_{\mathbf{2}}$ & $\mathbf{f}_{\mathbf{3}}$ & $\mathbf{f}_{\mathbf{d}}$ \\
\hline Fluid w/o structure & 1.2546 & 2.8761 & 4.0789 & 5.1344 \\
Structure w/o sloshing & 3.3628 & 16.4728 & 46.1918 & 94.4619 \\
Fluid with structure & 1.2413 & 2.8402 & 4.0904 & 5.1369 \\
Structure with sloshing & 3.4199 & 16.5525 & 46.1949 & 94.4664 \\
\hline
\end{tabular}

Table 9. Uncoupled and coupled fluid structure mode with or without fluid sloshing for $F_{D} \sim 1, M_{A}=8$ and $\lambda=50 \%$

\begin{tabular}{lcccc}
\hline \multicolumn{1}{c}{ Frequency $(\mathrm{Hz})$} & $\mathbf{f}_{1}$ & $\mathbf{f}_{2}$ & $\mathbf{f}_{3}$ & $\mathbf{f}_{4}$ \\
\hline Fluid w/o structure & 1.2905 & 2.8761 & 4.0789 & 5.1344 \\
Structure w/o sloshing & 2.7297 & 13.6706 & 40.4423 & 86.9110 \\
Fluid with structure & 1.2126 & 2.6420 & 4.1006 & 5.1410 \\
Structure with sloshing & 3.1352 & 13.6925 & 40.4485 & 86.9158 \\
\hline
\end{tabular}

Table 10. Uncoupled and coupled fluid structure mode with or without fluid sloshing for $F_{D} \sim 1, M_{A}=8$ and $\lambda=75 \%$

\begin{tabular}{lcccc}
\hline \multicolumn{1}{c}{ Frequency $(\mathrm{Hz})$} & $\mathbf{f}_{\mathbf{1}}$ & $\mathbf{f}_{\mathbf{2}}$ & $\mathbf{f}_{\mathbf{3}}$ & $\mathbf{f}_{4}$ \\
\hline Fluid w/o structure & 1.2962 & 2.8761 & 4.0789 & 5.1344 \\
Structure w/o sloshing & 1.9343 & 12.2546 & 37.7832 & 80.4374 \\
Fluid with structure & 1.0813 & 2.9832 & 4.0931 & 5.1385 \\
Structure with sloshing & 2.2147 & 12.3579 & 37.8024 & 80.4451 \\
\hline
\end{tabular}

Table 11. Uncoupled and coupled fluid structure mode with or without fluid sloshing for $F_{D} \sim 1, M_{A}=8$ and $\lambda=100 \%$

For a low dynamic FROUDE number, some fluid and structure modes are likely to be coupled. When an eigenfrequency of the structure coupled with a fluid without sloshing is near an 
eigenfrequency of fluid sloshing, a coupling effect can be observed. ${ }^{3}$ This coupling effect is characterized by the following facts:

$\checkmark$ the sloshing mode eigenfrequency is decreased when coupled with the structure;

$\checkmark \quad$ the structure mode eingenfrequency is raised because of the fluid added mass is lower due to sloshing effect;

$\checkmark$ the sloshing modes above the coupled one are increased whereas the sloshing modes under the coupled one are decreased.

In this case, an uncoupled analysis is not valid. The previous observations are more significant for a low mass number, as shown by Tab. (12).

\begin{tabular}{lcccc}
\hline \multicolumn{1}{c}{ Frequency $(\mathrm{Hz})$} & $\mathbf{f}_{\mathbf{1}}$ & $\mathbf{f}_{\mathbf{2}}$ & $\mathbf{f}_{\mathbf{3}}$ & $\mathbf{f}_{4}$ \\
\hline Fluid w/o structure & 1.2905 & 2.8761 & 4.0789 & 5.1344 \\
Structure w/o sloshing & 1.4055 & 8.0652 & 22.1989 & 45.7151 \\
Fluid with structure & 0.8375 & 3.0122 & 4.0972 & 5.1359 \\
Structure with sloshing & 2.0310 & 8.4110 & 22.2076 & 45.7264 \\
\hline
\end{tabular}

Table 12. Uncoupled and coupled fluid structure mode with or without fluid sloshing for $F_{D} \sim 1, M_{A}=1$ and $\lambda=75 \%$

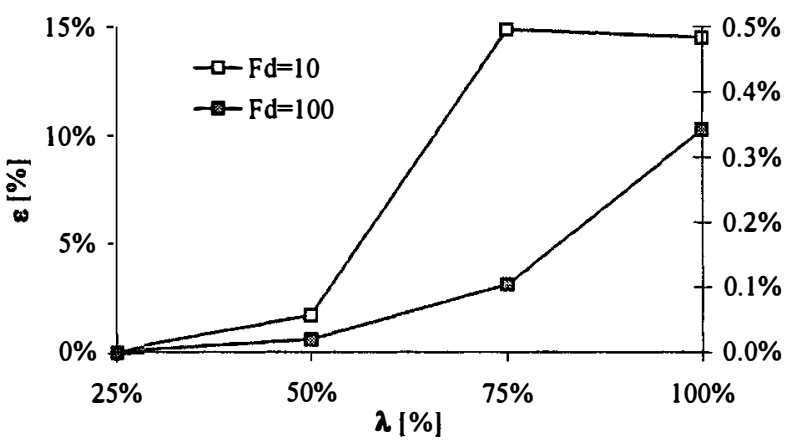

Figure 3. Evolution of the frequency ratio $\varepsilon$ of structure with or without fluid sloshing for different dynamic FROUDE numbers

Figure (3) gives the evolution of the ratio $\varepsilon=\frac{\omega_{w / o}-\omega_{w}}{\omega_{w / o}}$ between the first structure eigenfrequency coupled with the fluid with $\left(\omega_{w}\right)$ or without $\left(\omega_{w / o}\right)$ fluid sloshing the two dynamic FROUDE numbers toward the filling ratio $\lambda$, and shows the importance of the fluid sloshing for low FROUDE numbers and high filling ratio.

Figures (4) and (5) illustrates the coupling process for the case $F_{D}=10, \lambda=75 \%$.

\begin{tabular}{ccc}
\hline $\boldsymbol{\alpha}$ & Structure & Fluid \\
\hline 2 & $+14.86 \%$ & $-8.14 \%$ \\
5 & $+6.26 \%$ & $-2.40 \%$ \\
\hline
\end{tabular}

Table 13. Influence of confinement on the coupling effect

The influence of confinement on the coupling effect is given by Tab. (13), which compares the ratio $\varepsilon$ for the fluid and structure eigenfrequencies with or without sloshing (for the structure) and

\footnotetext{
${ }^{3}$ These effects of the coupling between fluid mode and structure mode in the case of a free surface have been observed by many authors for other fluid-structure coupled problems (see e.g. [3], and [15]).
}

with or without structure coupling (for the fluid), for two different values of $\alpha$. The coupling effect is amplified by confinement (i.e. for small values of $\alpha$ ).
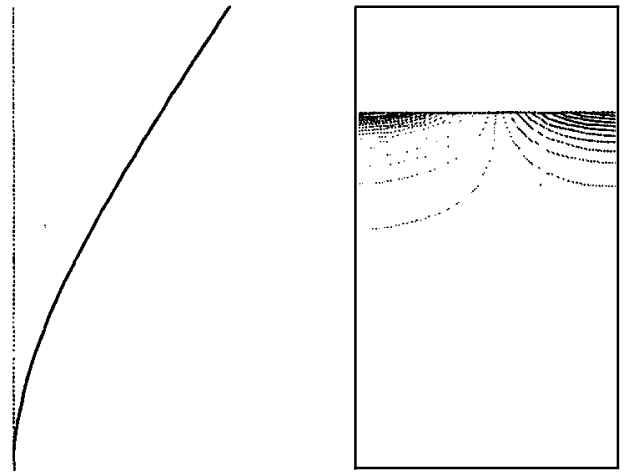

Figure 4. Structure mode without fluid $(3.4705 \mathrm{~Hz})$ and fluid sloshing mode without structure $(2.8761 \mathrm{~Hz})$
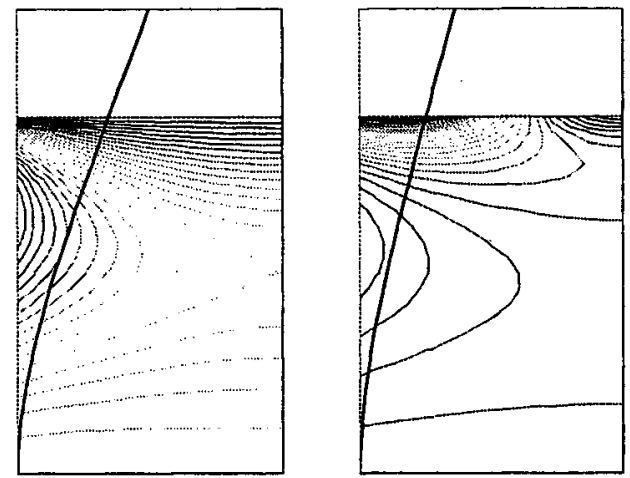

Figure 5. Structure mode coupled with fluid without fluid sloshing $(2.7297 \mathrm{~Hz})$ and with fluid sloshing $(3.1352 \mathrm{~Hz})$

When the fluid is supposed compressible, the coupled problem is represented by Eq. (14); due to the presence of the fluid mass matrix $M_{F}$, the global fluid mass matrix $M_{F}+M^{\prime}{ }_{F}$ is nonsingular, and a modal analysis can be performed on system given by Eq. (14), with the LANCZOS algorithm. As the study is related to low frequency phenomena, the matrix $M_{F}$ (e.g. the influence of the compressibility effects) has a slight influence on the numerical results, as shown by Tab. (14). The problem represented by Eq. (14) involves large scaled sparse matrices, whereas the problem represented by Eq. (16) deals with small scale filled matrices. Both systems are unsymmetric.

\begin{tabular}{lcccc}
\hline \multicolumn{1}{c}{ Frequency (Hz) } & $\mathbf{f}_{1}$ & $\mathbf{f}_{2}$ & $\mathbf{F}_{3}$ & $\mathbf{f}_{4}$ \\
\hline Fluid modes - Eq. (14) & 1.2905 & 2.8761 & 4.0789 & 5.1344 \\
Fluid modes - Eq. (17) & 1.2905 & 2.8761 & 4.0789 & 5.1344 \\
\hline Fluid coupled mode - Eq. (14) & 1.2126 & 2.6420 & 3.1352 & 4.1006 \\
Fluid coupled mode - Eq. (16) & 1.2126 & 2.6420 & 3.1352 & 4.1006 \\
\hline Structure coupled mode - Eq. (14) & 2.7297 & 13.671 & 40.442 & 86.911 \\
Structure coupled mode - Eq. (16) & 2.7297 & 13.670 & 40.441 & 86.905 \\
\hline
\end{tabular}

Table 14. Modal analysis with Eq. (14) or with Eq. (16)

The effects of coupling between fluid modes and structure modes can be represented in the following simple model with a 2D 
acoustic fluid and a single degree of freedom structure problem, as represented by Fig. (6).

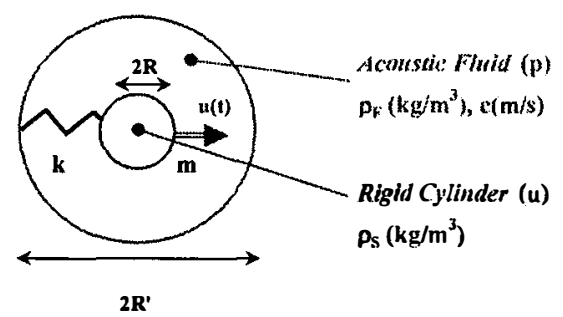

Figure 6. 2D fluid problem coupled with a single degree of freedom structure problem

$F(\omega)$ and $G(\omega)$ are defined by $G(\omega)=\frac{M_{A}}{\lambda(\alpha)} \times\left[\left(\omega_{0} / \omega\right)^{2}-1\right]$ and $F(\omega)=\frac{1}{\omega R / c} \times \frac{J(\omega R / c) Y^{\prime}(\alpha \omega R / c)-J^{\prime}(\omega R / c) Y(\alpha \omega R / c)}{J^{\prime}(\omega R / c) Y^{\prime}(\alpha \omega R / c)-J^{\prime}(\omega R / c) Y^{\prime}(\alpha \omega R / c)}$

where $J$ and $Y$ are the BESSEL functions of first kind and order one. One can show that the acoustic eigenpulsations are the roots of $\frac{1}{F(\omega)}=0$ and the structure eigenpulsation is the root of $G(\omega)=0$. The eigenpulsations of the coupled problem are roots of the equation

$$
F(\omega)=G(\omega)
$$

When the fluid is supposed uncompressible, $F(\omega)=1$. Equation (19) can be solved with Fig. (7), which plots $F$ and $G$ in the following cases.

$\checkmark$ Case \#1: the eigenfrequency of the structure is near an eigenfrequency of the fluid. Then, the structure eigenfrequency coupled with the fluid is greater in the case of a compressible fluid than in the case of an uncompressible fluid. The acoustic coupled modes are increased for modes above the coupled fluid/structure mode and decreased for other modes.

$\checkmark \quad$ Case \#2: the eigenfrequency of the structure is lower than all eigenfrequencies of the fluid. Then, the coupled eigenfrequency of the structure with the fluid is identical in the compressible and uncompressible cases. All the acoustic coupled eigenfrequencies are slightly increased, but the raise in frequency is significant for the first coupled acoustic modes.

$\checkmark$ Case \#3: the coupling effects are increase in the case of a low mass number.

The simple analytical model with acoustic and structure mode coupling can account for the observed coupling phenomena in the case of an elastic structure coupled with an uncompressible fluid with sloshing effects.

\section{DYNAMIC ANALYSIS}

A temporal analysis is performed on the structure problem, the fluid problem (i.e. with inner and outer cylinder considered as rigid walls) and the coupled fluid/structure problem, subjected to a sine wave shock.

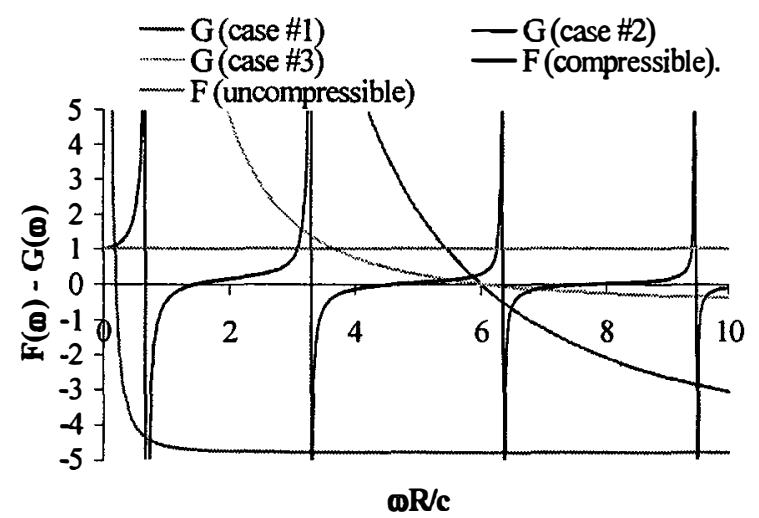

Figure 7. Graphical resolution of the elasto-acoustic problem of Fig. (6)

The imposed acceleration is given by:

$$
\gamma(t)=\left\{\begin{array}{c}
\gamma_{M} \sin \left(2 \pi \int_{o} t\right), \forall t \leq 1 / f \\
0, \forall t>1 / f
\end{array}\right.
$$

where $\tau=\frac{1}{f}$ is the shock duration. The discretized equation of motion for the structure, fluid or coupled fluid/structure problem are solved with the NEWMARK algorithm [10] with MATLAB.

\subsection{Structure problem}

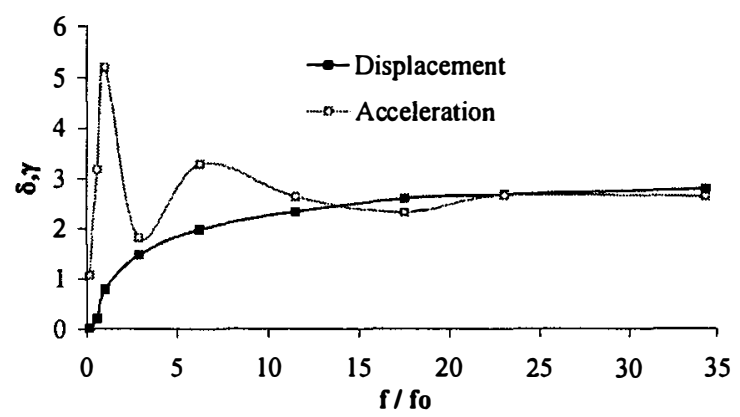

Figure 8. Reduced displacement and acceleration for the structure problem

Figure (8) gives a graphical representation of the reduced relative displacement and acceleration of the beam free end for various reduced shock frequencies, by plotting the mappings:

$f / f_{o} \mapsto \delta=\max \left(\frac{\ddot{Y}(h, t, f)}{\gamma_{M}}, \forall t \geq 0\right)$

and

$f^{\prime} f_{0} \mapsto \gamma=\max \left(\frac{Y(h, t, f)}{X_{M}}, \forall t \geq 0\right), \quad$ where $Z(h, t, f) \quad$ and

$\ddot{Y}(h, t, f)$ denotes the relative displacement and the acceleration of the beam free end. $\gamma_{M}$ and $X_{M}$ are the maximum imposed acceleration and displacement of shock with frequency $f$ and are linked according to: $X_{M}=\frac{\gamma_{M}}{2 \pi f^{2}} \cdot f_{o}$ is the first structure frequency. 
The curves are of course the same for the structure mass and stiffness corresponding to the two cases presented in Tab. (1): for a given problem (geometry and boundary conditions) the reduced displacement and acceleration only depend on the imposed motion [11].

\subsection{Fluid problem}

The dynamic equations of the fluid problem are discretized with finite element technique, as described in $\S .3$, to obtain the dynamic system:

$$
\left[M_{F}+M_{F}^{\prime}\right]\{\ddot{P}\}+\left[K_{F}\right]\{P\}=\left\{\Phi_{F}(\gamma)\right\}
$$

The fluid mass terms takes into account the sloshing and acoustic modes of the fluid.

The analytical solution of the uncompressible fluid problem with DIRICHLET conditions for the free surface is given by:

$$
\begin{aligned}
& p(r, \theta, z, t)= \\
& \frac{2 \rho_{F} \gamma(t)}{h} \cdot \times \sum_{n=0}^{n=+\infty}\left[\alpha_{n} I\left(q_{n} r\right)+\beta_{n} K\left(q_{n} r\right)\right] \cdot \frac{(-1)^{n} \cos \left(q_{n} z\right)}{g_{n}^{2}} \cdot \cos (\theta)
\end{aligned}
$$

and the fluid forces on the inner rigid cylinder is given by:

$$
\begin{aligned}
& \phi(t)=-\int_{z=0}^{z=h} \int_{\theta=0}^{\theta=2 \pi} p(R, \theta, z, t) R d \theta d z= \\
& -\left(\frac{2 \pi \rho_{F} R}{h} \cdot \sum_{n=0}^{n=+\infty} \frac{\left[\alpha_{n} I\left(q_{n} R\right)+\beta_{n} K\left(q_{n} R\right)\right]}{q_{n}^{3}}\right) \times \gamma(t)=-M_{H} \times \gamma(t)
\end{aligned}
$$

The added mass $M_{H}$ appearing on Eq. (23) characterizes the uncompressible behavior of the fluid, and is used to normalize the calculated fluid force.

Figure (9) gives the representation of the mapping $f / f_{o} \mapsto \varphi=\max \left(\frac{\Phi(t, f)}{\gamma_{M} M_{H}}, \forall t \geq 0\right)$ where $f_{o}$ is the first fluid sloshing frequency.

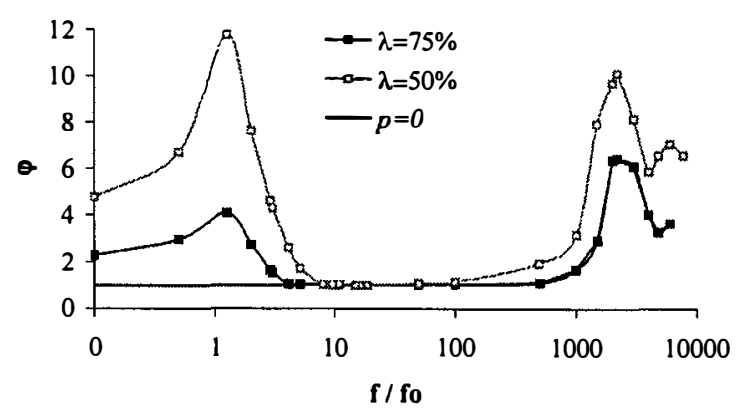

Figure 9. Reduced fluid force on the inner cylinder for the fluid problem

The curves exhibit resonance effects in the low (sloshing effects) and high (acoustics effects) reduced frequency range; in the medium frequency range, the fluid forces are governed by added mass effects.

\subsection{Coupled fluid/structure problem}

The discretization of the dynamic equations of the coupled problem with imposed motion leads to the following system, in which $U$ denotes the unknown displacement for the structure problem and $P$ represents the fluid pressure.

$$
\left[\begin{array}{cc}
M_{S} & 0 \\
-\rho_{F} R^{r} & M_{F}+M_{F}^{\prime}
\end{array}\right]\left\{\begin{array}{l}
\ddot{U} \\
\ddot{P}
\end{array}\right\}+\left[\begin{array}{cc}
K_{S} & R \\
0 & K_{F}
\end{array}\right]\left\{\begin{array}{l}
U \\
P
\end{array}\right\}=\left\{\begin{array}{c}
-m \ddot{X}-k X \\
\phi_{F}+\rho_{F} r \ddot{X}
\end{array}\right\}
$$

$X$ and $\ddot{X}$ are the imposed displacement and acceleration; $m$ and $k$ are extracted vectors of the structure global mass and stiffness matrices and $r$ is extracted of the global fluid/structure coupling matrix, taking onto account the imposed displacement [5]. The filling ratio is $\lambda=75 \%$.

Figure (10) shows the evolution of the reduced displacement of the structure coupled to the fluid without sloshing effects, i.e. only added mass effects are described.

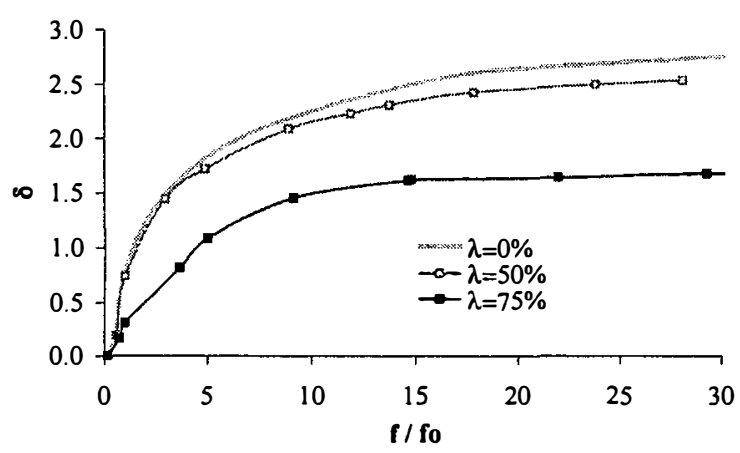

Figure 10. Reduced displacement for the coupled problem without fluid sloshing and various filling ratios

The reduced displacements are the same for the two FROUDE numbers. Applying a shock on both the structure and the fluid leads to an ARCHIMEDE fluid force that reduces the displacement of the structure when coupled with the fluid; this added mass effect is reduced for low filling ratios $(\lambda \leq 50 \%)$.

Figure (11) shows the evolution of the reduced displacement of the structure coupled to the fluid with sloshing effects for two dynamic FROUDE numbers. The shock frequency $f$ is reduced with the first frequency $f_{o}$ of the structure coupled with the fluid.

In the low reduced frequency range, the calculated displacement is higher with $F_{D}=10$ than $F_{D}=100$, due to coupling fluid sloshing and structure modes. In the medium and high frequency range, the computed displacement for the two dynamic FROUDE numbers are equivalent: pure added mass effects and acoustic effects have the same influence on the two systems.

Figure (12) compares the reduced displacement for the coupled problem with $F_{D}=10$, with and without fluid sloshing. 


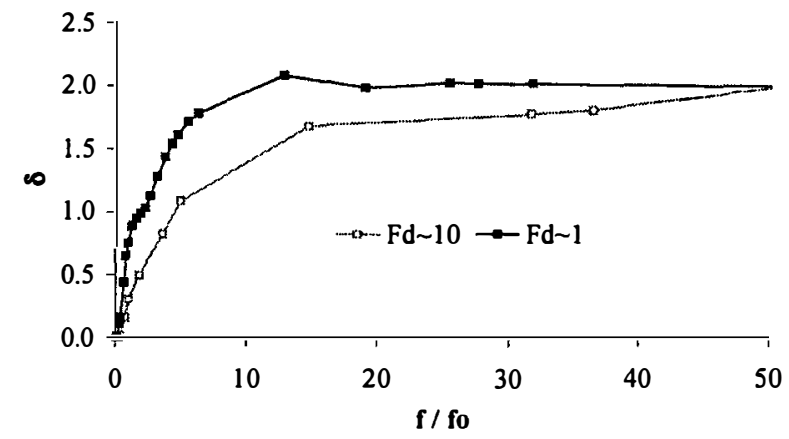

Figure 11. Reduced displacement for coupled problem with fluid sloshing in the case $F_{D} \sim 10$ and $F_{D} \sim 1$

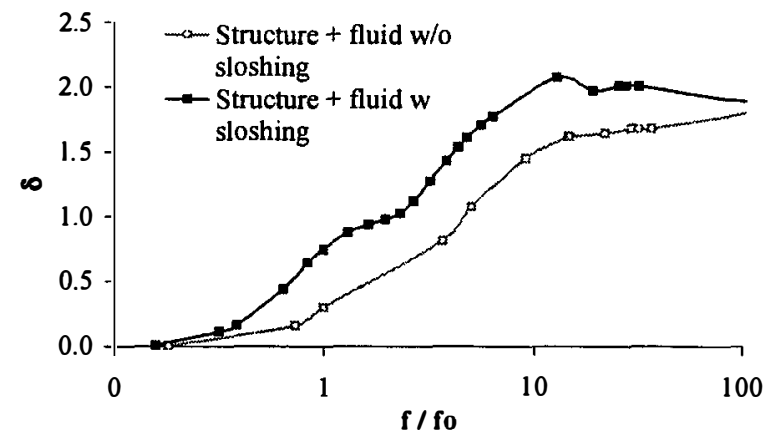

Figure 12. Reduced displacement for the coupled problem with and without fluid sloshing

It shows the importance of the coupling effect with sloshing mode for a low dynamic FROUDE number, since the calculated displacement of the coupled problem is greater when taking fluid sloshing into account. In both cases, the added mass (with or without compressibility) effects have the same influence.

\section{CONCLUSION}

A generic linear fluid structure coupled problem with fluid free surface is numerically studied by coupling fluid and structure finite element.

A modal and temporal analysis is performed on the coupled problem with or without taking into account the fluid gravity waves, for various dynamic Froude numbers defined with the geometrical and physical characteristics of the coupled problem.

For low dynamic Froude number, coupling effect between fluid sloshing modes and structure bending modes occur: the first frequencies of the coupled problem calculated with or without dynamic free surface effects can then be very different. The influence of other parameters such as the mass number and the confinement ratio is also exhibited on the coupling process.
A temporal analysis is also performed on the system, when subjected to a simple sine wave shock of given amplitude and duration.

Coupling effects are also illustrated by a comparison of the structure displacement under shock in various conditions.

Future study will focus on the non-linear sloshing effects by using a finite element and finite volume numerical coupling procedure.

\section{REFERENCES}

[1] K.C. Biswall, S.K. BhattachaKRya, P.K. Sinha. Coupled Dynamic Response of Liquid Filled Composite Cylindrical Tanks with Baffles. IMECE, New Orleans, 17-22 November 2002.

[2] R.D. BLEvins. Formulas for Frequency and Mode Shapes. R.E. Kreieger Publishing Company, 1984.

[3] J.R. CHO, J.M. SONG. Assessment of Classical Numerical Models for the Separate Fluid-Structure Modal Analysis. Journal of Sound and Vibration, 239, 995-1012, 2001.

[4] C. Conca, J. Planchard, M. Vanninathan. Existence and location of Eigenvalues for Fluid-Solid Structures. Computer Methods in Applied Mechanics and Engineering, 77, 253-291, 1989.

[5] S. DUBIGEON. Mécanique des milieux continus. Lavoisier, 1998.

[6] J.B. FrandSEN, A.G.L BORTHWICK. Non Linear Sloshing in Fixed and Vertically Excited Containers. IMECE, New Orleans, 17-22 November 2002.

[7] R.J. FRITZ. The Effect of Liquids on the Dynamic Motion of Immersed Solids. Journal of Engineering for Industry, 167-173, 1972.

[8] R.J. GIBERT. Vibration des structures. Interaction avec les fluides. Sources d'excitation aléatoires. Collection de la Direction des Etudes et Recherches d'Electricté de France, vol. 69, Eyrolles, 1986.

[9] G.H. GolUB, C.F. VAN LOAN. Matrix Computation. Johns Hopkins University Press, 1994.

[10] T.J.R Hughes, T. BelytschKo. A Précis of Developments in Computational Methods for Transient Analysis. Journal of Applied Mechanics, 50, 1033-1041, 1983.

[11] C. LaLANNE. Vibrations et choc mécaniques. Hermès, 1999.

[12] E. DE LANGRE. Fluides et Solides. Les éditions de l'Ecole Polytechnique, 2002.

[13] J. MAKERLE. Fluid-Structure Interaction Problems, Finite Element Approach and Boundary Elements Approaches. A Bibliography. Finite Elements in Analysis and Design, 31, 231-240, 1999.

[14] H. J.P. MORAND, R. OHAYON. Fluid Structure Interaction. Wiley \& Sons, 1995.

[15] B. PESEUX, A. PIARD. Dynamic response of immersed structures in bounded domain with gravity waves. ASME-Pressure Vessel and Piping, San Diego, 26-30 July 1998.

[16] C. RAJAKUMAR, C.G. Rogers. The Lanczos Algorithm Applied to Unsymmetric Generalized Eigenvalue Problem. International Journal of Numerical Methods in Engineering, 32, 1009-1026, 1991.

[17] J.S. SCHOTTÉ, R. OHAYON. Effects of Gravity on a Free Surface FluidStructure System. IMECE, New Orleans, 17-22 November 2002

[18] J.F. Sigrist, C. LAINÉ, D. LemoIne, B. PESEUX. Choice and Limits of a Linear Fluid Model for the Numerical Study in Fluid Structure Interaction Problem. Pressure Vessel and Piping, Cleveland, 20-24 July 2003.

[19] J.F. Sigrist, V. Melot, C. Laine, D, B. PeseuX. Numerical Simulation of Fluid Structure Problem by Coupling Fluid Finite Volume and Structure Finite Element or Modal Approach. Flow Induced Vibration, Paris, 4-8 Juillet 2004.

[20] I.N. SNEDDON. Encyclopaedic Dictionnary of Mathematics for Engineers and Applied Scientists. Pergamon, 1976. 